\title{
Analysis of the Causes of Chronic Pain after Inguinal Hernia Repair without Tension and Its Prevention and Treatment
}

\author{
Hao Wu${ }^{1}$, Weimin $\mathrm{Li}^{2 *}$ \\ ${ }^{1}$ School of Medicine, Wuhan University of Science and Technology, Wuhan, China \\ ${ }^{2}$ Xiaogan Hospital Affiliated to Wuhan University of Science and Technology, Wuhan University of Science and Technology, \\ Xiaogan, China \\ Email: 502559545@qq.com, *xglwm1962@163.com
}

How to cite this paper: $\mathrm{Wu}, \mathrm{H}$. and $\mathrm{Li}$, W.M. (2019) Analysis of the Causes of Chronic Pain after Inguinal Hernia Repair without Tension and Its Prevention and Treatment. International Journal of Clinical Medicine, 10, 122-127.

https://doi.org/10.4236/ijcm.2019.103012

Received: January 30, 2019

Accepted: March 4, 2019

Published: March 7, 2019

Copyright $\odot 2019$ by author(s) and Scientific Research Publishing Inc. This work is licensed under the Creative Commons Attribution International License (CC BY 4.0).

http://creativecommons.org/licenses/by/4.0/

\begin{abstract}
Purpose: Analyzing the causes of pain after tension-free repair in the inguinal hernia, and propose corresponding treatment strategies. Results: The patients in this group were followed up for $3-12$ months. A total of 5 patients still had pain relief within 3 months after surgery. Further treatment was needed, and the incidence rate was $5 \%$. Conclusion: Skilled surgical procedures are performed in patients with local anesthesia, and some of the absorbable repair materials can be used. The improved patch fixation and extra peritoneal repair can reduce the pain after inguinal hernia repair without tension.
\end{abstract}

\section{Keywords}

Inguinal Hernia, Tension-Free Hernia Repair, Chronic Pain, The Reason, Treatment

\section{Introduction}

Inguinal hernia is a common disease in general surgery and can be cured by surgery. Inguinal hernia repair is one of the most common surgical procedures [1]. Worldwide, approximately 20 million patients undergo inguinal hernia repair each year [2]. According to reports [3], in patients undergoing inguinal hernia repair, $16 \%$ to $62 \%$ have postoperative chronic groin pain. Although this pain is usually mild in nature, studies on the quality of life of patients with chronic pain in the inguinal hernia indicate that the severity of chronic pain can seriously affect normal daily activities. After extensive patch repair, the recurrence rate after inguinal hernia surgery has been accepted, and the focus of the 
study has shifted from recurrence to chronic pain. Although pain can be controlled with analgesics, postoperative chronic pain remains a major clinical concern and significantly affects the patient's quality of life [4].

\section{Definition of Postoperative Chronic Pain}

Persistent pain: defined as at least the presence of pain, cannot be ignored, and interferes with daily activities of the past week Persistent pain: defined as at least the presence of pain, cannot be ignored, and interferes with daily activities of the past week [5]. Since the publication of Chronic postsurgical pain (CPSP), the current postoperative chronic pain has gradually received the attention of clinicians. However, CPSP does not have a complete and definitive definition. It generally refers to persistent pain that occurs after surgery. A large number of literatures are based on postoperative pain for 3 months. It is also believed that CPSP refers to a situation in which postoperative pain is unlikely to be relieved, difficult to handle, and pain is still exceeded beyond normal recovery time. Zhou Jianping [6] believes that although the International Association for the Study of Pain has determined that symptoms last for more than 3 months as chronic pain, in actual sputum surgery, the chronic inflammatory response lasts for more than 3 months due to the implantation of foreign bodies such as patches. Therefore, some people have pain symptoms for 6 months or longer as the standard for postoperative chronic pain. Zhou Jianping [7] said in his case report that chronic pain after tension-free hernia repair persisted for more than 3 months after surgery, and the pain range exceeded the pain in the affected area. It is a common complication after hernia repair. The incidence rate varies widely from literature to literature, ranging from about $5 \%$ to $35 \%$. Since the inflammatory reaction of foreign substances such as patches can last for several months, the pain is currently more than 6 months as the standard for postoperative chronic pain. Therefore, the author believes that the tracking time of postoperative chronic pain should be 3 months or 6 months after surgery, because the incidence of chronic pain will decrease correspondingly with the extension of time, so the selected postoperative study The time points are different, so the incidence of chronic pain is also different.

\section{Pain Mechanism}

The pathophysiological mechanisms of chronic pain are complex and depend on the type of pain, including somatic, neurological, non-neuro (inflammatory non-neural pain) and visceral pain [8]. Somatic pain is sometimes referred to as periostitis and is confined to the pubic tuberosity, usually due to damage to the periosteum of the pubic tuberosity. Neuralgia is thought to be caused by damage to the inguinal nerve, causing pain in the sensory distribution of the affected nerve. The most common are the genital branches of the inguinal hernia, the inferior epigastric nerve, and the genital femoral nerve. For laparoscopic surgery, the femoral or femoral cutaneous nerves of the inferior femoral artery may also 
be involved. Nerve damage can occur during or after surgery, and the mechanisms include indirect or direct structural damage and nerve crush injuries, caused by sutures or fixation devices, folded mesh, or inflammation and scarring around the nerves. Non-neuropathic pain is the result of tissue damage and local inflammatory response, caused by endogenous inflammatory mediators acting on pain receptors. Visceral pain is pain that is felt in the gut, spermatic cord, or other structures surrounding the urethra. Zhou Jianping believes that pain may be related to the following three aspects: one is the direct injury to the nerve in the surgical area during surgery; the other is the deformation and dysfunction of the nerve structure caused by scar contraction and deformation during postoperative tissue healing; Psychological factors, patients pay too much attention to this local discomfort, leading to serious psychological reactions, may be related to the reduction of pain threshold caused by nerve remodeling [6]. The most important of these is nerve damage. The mechanism may be caused by sensory and motor neurological disorders in the groin area. The nerves associated with pain are mainly the inferior tibia, the inguinal hernia, and the reproductive nerve [7]. Nerve ligature or suture, mesh scar tissue compression nerve, neuroma formation, etc. are the main causes of chronic pain after inguinal hernia [7].

\section{Materials and Methods}

\subsection{Capital Information}

100 patients with inguinal hernia who underwent tension-free repair between 2015 and June 2017 were selected as males. The age ranged from 21 to 79 years, with an average of 64.2 years. Among them, 75 cases were inguinal hernia, 22 cases were inguinal hernia, and 3 cases were compound hernia. Among them, 10 cases were bilateral inguinal hernia, and 8 cases recurred after inguinal hernia.

\subsection{Method}

\subsubsection{Surgical Methods}

Forty-five patients were treated with a tapered ankle ring filler and patch (manufactured by Bard, USA), 22 patients were treated with a clinical conical ankle ring filler (for domestic phase III), and 23 patients were treated with partially absorbable materials (Produced by Johnson \& Johnson, USA). Twenty patients underwent local anesthesia and 80 patients underwent continuous epidural anesthesia or spinal anesthesia. Twenty-six patients underwent plain patch repair, 70 underwent iliac ring-filled tension-free repair, and 4 underwent plain laparoscopic repair.

\subsubsection{Pain Management Strategy}

First, patients should be given local physiotherapy such as infrared light and acupuncture. Analgesic drugs should be applied to patients with unresolved pain, mainly non-steroidal drugs. For patients with acute neuropathic pain, nerve block therapy can be applied. Dexamethasone and 0.5 can be used. \% li- 
docaine was treated with local block injection, once/week for 3 - 5 weeks. The inguinal, inguinal, and femoral nerves were injected around the femoral nerve. The site of tenderness was often used. Surgical treatment is feasible after non-surgical treatment of pain.

\section{Result}

The patients were followed up for 3 - 13 months. A total of 5 patients still had pain relief at 3 months after surgery. Further treatment was needed, with a $5 \%$ incidence. Among them, 1 case of skin redness/local pain was considered to be a chronic inflammatory reaction caused by materials. Symptoms disappeared after 5 days of treatment with antibiotics and hormones, swelling and discomfort occurred around 2 incisions, tenderness at the upper end of the incision, symptoms after drug treatment and physiotherapy Relieved or disappeared, 2 cases of pubic nodules to the upper part of the scrotum and upper thigh skin pain, symptoms disappeared after 3 - 5 times of nerve block treatment.

\section{Discussion}

The use of tension-free hernia repair for inguinal hernia has the advantages of short recovery time, low recurrence rate and few complications. At present, clinicians mainly use this surgical method to treat inguinal hernia, but the incidence of postoperative pain is higher. About $5 \%$ to $35 \%$, in addition to the recurrence of inguinal hernia, another major problem that seriously plagues them. This article summarizes the causes of postoperative pain as follows.

\subsection{Repair Material Factor}

Polypropylene mesh and filler are more repair materials used at the present stage. Although their tissue compatibility is good, the surface is rough, the texture is hard, and it is easy to form fiber adhesion or scar with the surrounding tissue, while the tapered filler is often A fibrous mass is formed that compresses the surrounding tissues and nerves, producing a distinct foreign body sensation and pain. Chronic rejection and inflammatory responses to the material can also cause pain.

\subsection{Fixed Factor of Material}

When a good patch fixation is not performed, the patch is displaced, twisted, or shrunk, and the surrounding blood vessels and nerves are compressed to cause pain. If the patch is fixed by a method of fixing the thread by one turn, the patient may cause foreign body repulsion, which may cause infection, and the patch may be tied to the nerve when the patch is excessively fixed, so that postoperative pain occurs. In addition to the appeal factors, the compression and separation of the spermatic vessels in the surgery is too extensive, the formation of hematoma in the patient's incision and related psychological factors often cause postoperative pain in patients. 
According to the cause of postoperative pain, the following aspects should be noted during surgery: 1) Surgery should be performed by a physician with clinical experience in the Department of Surgery. Sharp separation should be used during the operation. Care should be taken to protect the lower abdomen, the groin and the reproductive femoral nerve. When the cremaster muscle is cut and the inner ring is dissected, the reproductive nerve is avoided. The reproductive branch causes damage. Avoid deep sutures at the pubic nodules to prevent periosteal traction. Preperitoneal repair of plain films can reduce the rate of nerve damage, thereby reducing the incidence of postoperative pain. In this study, 4 patients were treated with preperitoneal repair, and no pain occurred after operation. 2) Individualized solutions should be used when using materials. If the patient is thin or has a small ankle ring, the plain film can be used alone. If the patient's financial situation is available, some absorbable materials can be used. Because the material is soft, the surrounding area can be lightened. The inflammatory response of the tissue is better. In this study, 23 patients with partial absorbable patches did not have postoperative pain. 3) When the repair material is fixed, the hospital uses absorbable sutures, which can significantly reduce the foreign body rejection of the suture and the infection caused by the suture, and can avoid the postoperative pain. In recent years, medical adhesives have been used to fix the patch, so that the pain caused by the occlusion of the nerve has been avoided, and the effect is good, and the patient has no pain after the operation. 4) During the operation, the spermatic cord should be protected and the blood should be carefully stopped to prevent hematoma from forming. If the patient's isolated wound is large, a drainage tube should be placed to reduce the occurrence of scrotal swelling. In this study, 3 of the 5 patients with pain were recurrent sputum. Considering the psychological factors of the patients, they could be given psychological intervention.

\subsection{Neurological Factor}

Neurological factors are the main cause of postoperative pain and are considered as a result of groin movement and sensory neurological disorders. The genital nerve of the inferior tibia, the inguinal hernia and the genital tract are the main nerves. Because of the large variation, the recurrence after the operation of the sputum can lead to anatomical unclear, which is easy to cause damage to the nerve during surgery and form a neuroma. There is severe pain that cannot be alleviated. And the pubic symphysis periosteum is rich in nerve fibers. When the patch is fixed, it often causes severe pain after the needle is inserted in the periosteum, which causes the patient to have a more obvious tingling sensation when walking the leg after the operation.

In the treatment of inguinal hernia after tension-free repair, the hospital believes that targeted treatment should be taken according to the actual situation of the patient. If the patient's symptoms are mild and intermittent pain, then the drug treatment and physiotherapy can be used to receive significant therapeutic 
effects. The drugs mainly use weak opioid analgesics and non-steroidal drugs; If the patient has an early rejection or chronic inflammatory response, antibiotics and hormonal drugs should be used; If the patient has neurological involvement, local nerve block should be performed. Local anesthetic plus steroid mixture should be used for treatment. The main nerves around the main inguinal region such as the inguinal, groin and reproductive femoral nerve are the main injection sites. The patient with obvious trigger point can be injected at the trigger point; If the patient's pain cannot be alleviated after taking the above measures, the patch material should be taken out.

\section{Conclusion}

In summary, skilled surgical procedures in patients with local anesthesia, the use of partial absorbable repair materials, improved patch fixation and extraperitoneal repair can make pain in the inguinal hernia without tension repair cut back.

\section{Conflicts of Interest}

The authors declare no conflicts of interest regarding the publication of this paper.

\section{References}

[1] Kingsnorth, A. and Leblanc, K. (2003) Hernias: Inguinal and Incisional. Lancet, 362, 1561-1571. https://doi.org/10.1016/S0140-6736(03)14746-0

[2] Öberg, S., Andresen, K., Klausen, T.W., et al. Chronic Pain after Mesh versus Nonmesh Repair of Inguinal Hernias: A Systematic Review and a Network Metaanalysis of Randomized Controlled Trials. Surgery.

[3] Jeroukhimov, I., Wiser, I., Karasic, E., et al. (2014) Reduced Postoperative Chronic Pain after Tension-Free Inguinal Hernia Repair Using Absorbable Sutures: A Singleblind Randomized Clinical Trial. Journal of the American College of Surgeons, 218, 102-107. https://doi.org/10.1016/j.jamcollsurg.2013.09.010

[4] Nikkolo, C. and Lepner, U. (2015) Chronic Pain after Open Inguinal Hernia Repair. Postgraduate Medicine, 128, 69. https://doi.org/10.1080/00325481.2016.1121090

[5] Lundström, K.J., Holmberg, H., Montgomery, A., et al. (2017) Patient-Reported Rates of Chronic Pain and Recurrence after Groin Hernia Repair. British Journal of Surgery, 105. https://doi.org/10.1002/bjs.10652

[6] Zhou, J.P. and Shu, G.S. (2009) Chronic Pain after Inguinal Hernia Repair. Chinese Journal of Abdominal and Abdominal Surgery (Electronic Edition), 3, 371-375.

[7] Ren, F. and Zhou, J.P. (2011) Treatment of Chronic Pain after Tension-Free Repair of Inguinal Hernia with Nerve Ablation and Mesh Removal: Report of One Case. Chinese Journal of Modern Surgery, 15, 441-442.

[8] Hu, Q.L. and Chen, D.C. (2018) Approach to the Patient with Chronic Groin Pain. Surgical Clinics of North America. https://doi.org/10.1016/j.suc.2018.02.002 